\title{
Multipath detection with the combination of SNR measurements - Example from urban environment
}

\author{
Peter Špánik*, Ján Hefty \\ Slovak University of Technology in Bratislava \\ Faculty of Civil Engineering \\ Department of Theoretical Geodesy \\ Radlinského 11, 81005 Bratislava, Slovakia \\ e-mails: peter.spanik@stuba.sk; jan.hefty@stuba.sk; \\ * Corresponding author: Peter Špánik
}

Received: 17 July / Accepted: 14 September 2017

\begin{abstract}
Multipath is one of the most severe station-dependent error sources in both static and kinematic positioning. Relatively new and simple detection technique using the Signal-to-Noise (SNR) measurements on three frequencies will be presented based on idea of Strode and Groves. Exploitation of SNR measurements is beneficial especially for their unambiguous character. Method is based on the fact that SNR values are closely linked with estimation of pseudo-ranges and phase measurements during signal correlation processing. Due to this connection, combination of SNR values can be used to detect anomalous behavior in received signal, however some kind of calibration in low multipath environment has to be done previously. In case of multipath, phase measurements on different frequencies will not be affected in the same manner. Specular multipath, e.g. from building wall introduces additional path delay which is interpreted differently on each of the used carrier, due to different wavelengths. Experimental results of multipath detection in urban environment will be presented. Originally proposed method is designed to work with three different frequencies in each epoch, thus only utilization of GPS Block II-F and Galileo satellites is possible. Simplification of detection statistics to use only two frequencies is made and results using GPS and GLONASS systems are presented along with results obtained using original formula.
\end{abstract}

Keywords: multipath, detection, SNR, three-frequency combination, GNSS positioning

\section{Introduction}

Signals of GNSS satellite's constellation follow the same general laws of propagation like any other electromagnetic signals. Effects like signal attenuation, reflection, refraction or scattering occur how the signal travels from satellite transmitting antenna through physical space to receiving antenna. In the urban environment, 
where buildings and other structures obscure clear sky view, many reflections can be expected. As the GPS Interface Control Document stated (IS-GPS-200, 2013), all GPS satellites transmit signals with right-hand circular polarization (later abbreviated as RHCP). Also other GNSS constellations use RHCP signals because of its beneficial property in case of signal reflection when incident RHCP signal largely changes its polarization state to left-hand circular polarization (LHCP). Along with a change of polarization, the signal attenuation occurs and thus reflected signal is always less powerful than incident signal. Power of reflected signal can be described by reflection coefficients (sometimes referred as Fresnel coefficients), which depends on material reflection properties and signal incident angle. Material is for this purpose defined by physical constants $\mu$ (magnetic permeability) and $\varepsilon$ (electric permittivity). For more details on signal reflection and other effects concerning with signal propagation see (Richards, 2008). Polarization change of direct RHCP signal to LHCP during reflection is not done perfectly. As it is described in a paper of (Lau and Cross, 2007) polarization efficiency is always less than $100 \%$. As a result of non-ideal polarization change, or due to two consecutive reflections, reflected signal can interact with direct RHCP signal. This disturbing effect of signal reflection is commonly known as GNSS multipath. Most antennas used for geodetic surveys are intended to effectively receive only RHCP signals and reject LHCP signals. In this way antennas highly reduce the multipath effect's impact even before that receiver process signal. Unfortunately there is still residual portion of RHCP reflected signal which can interfere with direct signal and subsequently composed signal is tracked instead of direct one. Composed signal has different amplitude and the phase than the direct one as it can be clearly seen in the Figure 1.

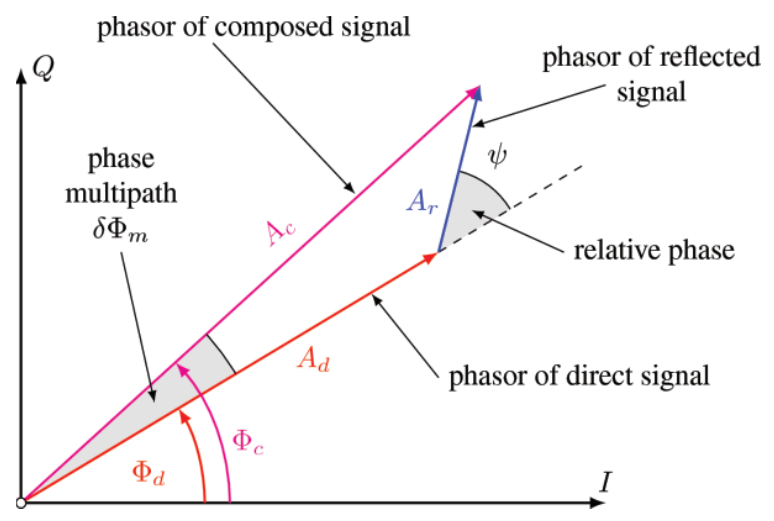

Fig.1. In-phase (I) and Quadrature phase (Q) components of direct, reflected and composed signal as seen during carrier phase determination.

Letter $A$ represents amplitude and $\Phi$ the phase of the corresponding phasor. Amplitude of reflected signal $A_{r}$ is defined by material properties and incident angle. 
Amplitude of composed signal during carrier phase determination describes received signal strength. The receiver during process of phase lock loop tracking correlates the incoming signal with a signal replica. Side product of this process is the signalto-noise ratio denotes as SNR. Normally, SNR measurements are obtained using the signal and noise power of the modulated signal at the correlator output (Luo, 2013). SNR values are originally computed in $\mathrm{dB}$ scale for specific receiver processing bandwidth. SNR in this form could be slightly misleading because the processing bandwidth is often unknown. More convenient way is to store SNR values in units of $\mathrm{dBHz}$ (also denotes as $\mathrm{dB}-\mathrm{Hz}$ ) which relate signal-to-noise per $1 \mathrm{~Hz}$ bandwidth (Hofmann-Wellenhof et al., 2008). Nowadays dBHz unit is widely used amongst receiver's manufacturers to record values of SNR measurements.

If we assume that SNR measurements are proportional to amplitude of composed signal, then by application of cosine law on triangle in Figure 1 and we can write following equation (1):

$$
S N R \sim A_{c}^{2}=A_{d}^{2}+A_{r}^{2}+2 A_{d} A_{r} \cos \psi
$$

This equation shows how parameters of reflected signal $\left(A_{p} \psi\right)$ influence multipath contaminated SNR values. As it is schematically shown in (Lau and Cross, 2006), maximal differences of SNR values compared to its nominal values (corresponding only to direct signal) is when relative phase $\psi=0^{\circ}$ or $\psi=180^{\circ}$. On the other hand SNR have very similar values to its nominal ones if relative phase is $\psi=90^{\circ}$ or $\psi=270^{\circ}$. Probably the most important conclusion from this point of view is that phase multipath $\delta \Phi_{\mathrm{m}}$ can be linked directly to multipath contaminated SNR values. In (Lau and Cross, 2006) authors conclude that there is approximately $90^{\circ}$ phase difference between multipath contaminated SNR values and phase multipath error. This fact can be used to introduce some form of SNR-based stochastic modeling as the Lau and Cross suggested or it can be used to directly correct multipath contaminated phase measurements as it shown in (Rost and Wanninger, 2009). In this case position precision was improved by $25 \%$ after application of simplified phase correction.

Relative phase of reflected signal $\psi$ plays important role in description of phase multipath as it is briefly described above. Relative phase $\psi$ we can imagine simply like reminder of path delay $\Delta d$ after subtraction of integer number $n$ of wavelengths $\lambda_{i}$ at carrier frequency $i$. We have to deal with angular and metric units to correctly express $\psi_{i}=\left(\left(\Delta d-n \lambda_{i}\right) / \lambda_{i}\right) \cdot 360^{\circ}$ in degrees. If we compute relative phases for different carriers (e.g. L1, L2, L5 for GPS) they will be different, because of not equal carrier wavelength. Because of different relative phases there will be subsequently also different effect on multipath error and also SNR values at each carrier frequency. 


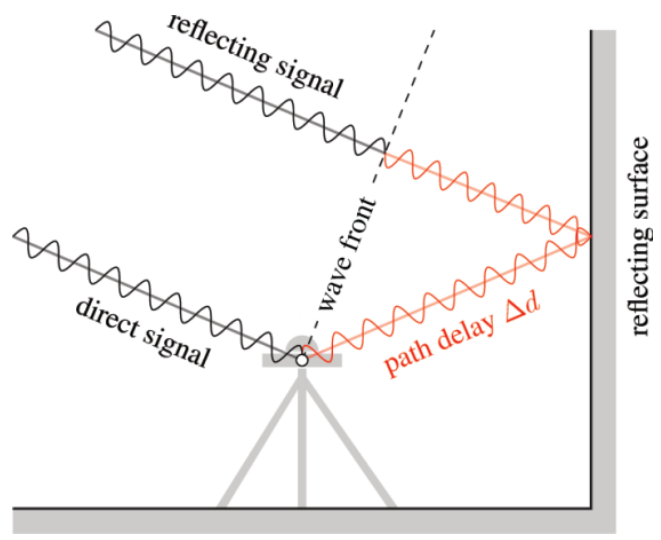

Fig. 2. Example of specular reflection. Path delay $\Delta d$ is expressed as difference between path of reflecting signal ray and direct signal ray. Wave front is also shown to highlight path delay.

\section{Methodology}

Finding that the presence of phase multipath has a different impact on SNR measurements at each of tracked carrier frequency, exploded Strode and Groves in (Strode and Groves, 2016). In general terms they proposed a detection algorithm which computes a test statistic from a set of current SNR measurements and then compares it with threshold that marked the limit of the system's normal performance. Exceeding test statistic over the threshold indicates the presence of multipath. SNR measurements at three different carrier frequencies are used to compute test statistic $S_{a}^{s}$, thus only utilization of GPS Block II-F and Galileo satellites is possible:

$$
S_{a}^{S}=\sqrt{\left(S N R_{a}^{s, L 1}-S N R_{a}^{S, L 2}-\Delta \hat{C}_{12}\left(\theta_{a}^{S}\right)\right)^{2}+\left(S N R_{a}^{s, L 1}-S N R_{a}^{S, L 5}-\Delta \hat{C}_{15}\left(\theta_{a}^{S}\right)\right)^{2}}
$$

where $S N R_{a}^{S, L 1}$ denotes SNR measurement on GPS L1 carrier frequency between antenna $a$ and satellite $s, \Delta \hat{C}_{12}$ and $\Delta \hat{C}_{15}$ are predicted L1 - L2, respectively L1 - L5 SNR differences acquired in low-multipath environment modeled as a function of satellite elevation $\theta_{a}^{s}$. Equation of test statistic (2) can be simplified to use only two frequencies as (3). This could be more susceptible to incorrect multipath identification because of less robustness, but will allow usage of GLONASS system with only two available frequencies.

$$
S_{a}^{S}=\left|S N R_{a}^{S, L 1}-S N R_{a}^{S, L 2}-\Delta \hat{C}_{12}\left(\theta_{a}^{S}\right)\right|
$$




\subsection{Calibration measurement}

System normal performance need to be determined in low-multipath environment, in different words in environment where no objects are in close vicinity of antenna and no reflections are expected. This measurement campaign we can name as calibration measurement, because it will further represent reference for multipath detection. For this purpose an open field with low vegetation grow has been chosen, as it is visible in Figure 3. Measurement took place near Bratislava and lasted approximately 8 hours. Combined geodetic GNSS receiver Trimble R8 Model 3 was used with tracking capability of GPS, Galileo and GLONASS systems at $1 \mathrm{~s}$ sampling. Totally 24 GPS satellites (8 GPS Block II-F) and 10 GLONASS satellites were used to estimate polynomial coefficients of $\Delta \hat{C}_{12}$, respectively $\Delta \hat{C}_{15}$ differences as a function of satellite elevation. Only data with elevation above 10 degrees were used. Linear and parabolic trend function $\Delta \hat{C}$ has been found as sufficient to appropriate fit measured data. Data fit is shown on Figure 4. In case of GPS three frequency (Figures 4a, 4b) and two frequency (Figure 4c) approaches were computed. Numerical characteristics of fitted $\Delta \hat{C}$ curves for all approaches according to (2) or (3) are listed in Table 1. Values are antenna and receiver dependent and have to be estimated separately for different equipment. In case of modular system, calibration coefficients depend also on antenna cable length.

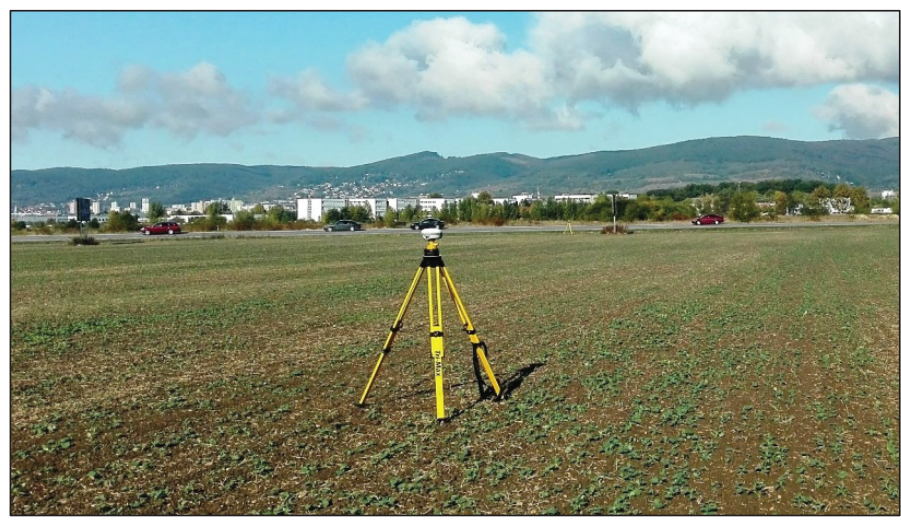

Fig. 3. Combined Trimble R8 Model 3 GNSS receiver setup in low-multipath environment during calibration measurement. Measurement took place near Bratislava - Vajnory, Slovakia on 7 October, 2016. 

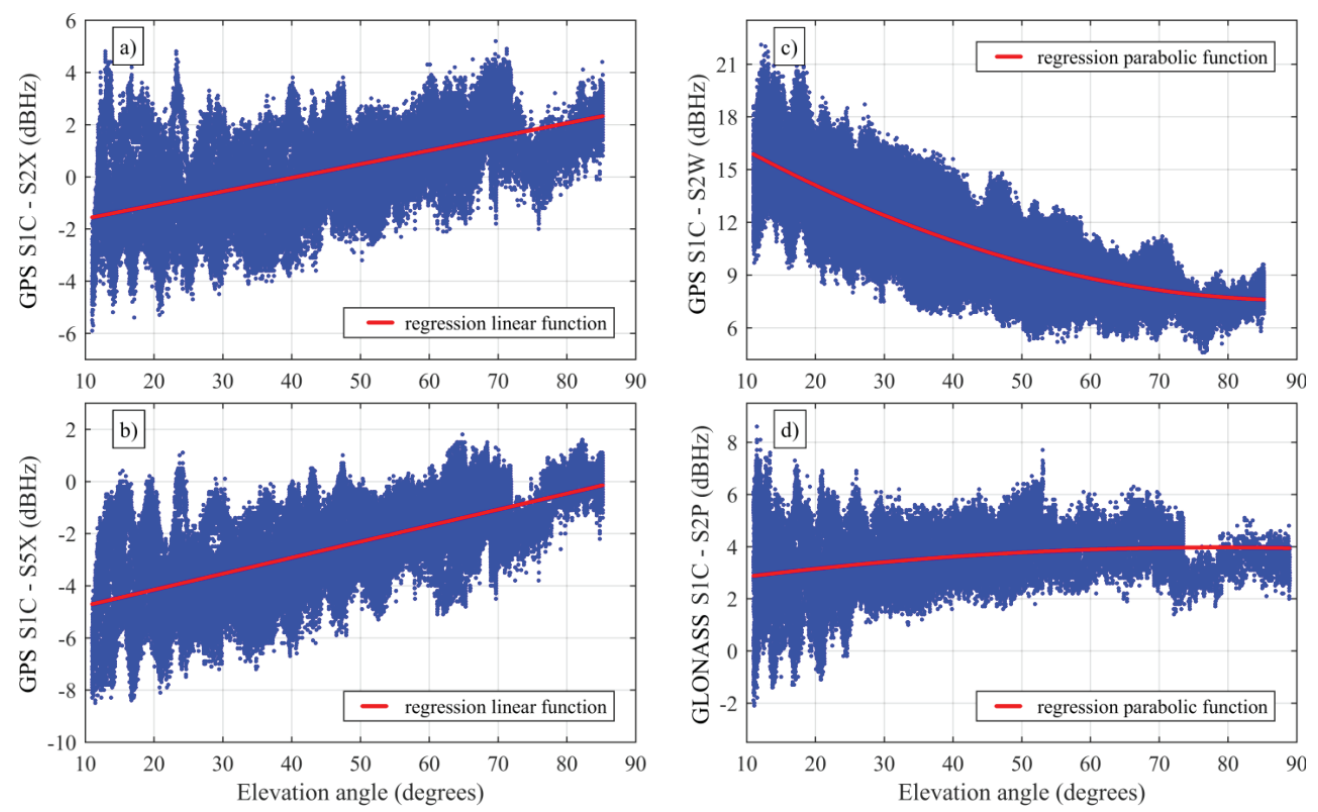

Fig. 4. Differences of calibration SNR measurements: a), b) are GPS SNR differences to compute $S_{a}^{s}$ using (2), c) GPS, respectively d) GLONASS SNR differences to compute $S_{a}^{S}$ by (3). In case of a), b) linear regression function was used and in c), d) parabolic function was used.

Table 1. Estimated coefficients of $\Delta \hat{C}$ regression function for different approaches

\begin{tabular}{|l|l|l|l|l|}
\hline \multirow{2}{*}{ System } & Approach & SNR combination & Coefficients of $\Delta \hat{C}$ & $\Delta \hat{C}$ fit RMS \\
\hline \multirow{3}{*}{ GPS } & \multirow{2}{*}{3 -freq. } & S1C, S2X & $\begin{array}{l}a=-2.13 \mathrm{dBHz} \\
b=0.05 \mathrm{dBHz} / \mathrm{deg}\end{array}$ & $1.33 \mathrm{dBHz}$ \\
\cline { 2 - 5 } & \multirow{2}{*}{ 2-freq. } & S1C, S5X & $\begin{array}{l}a=-5.38 \mathrm{dBHz} \\
b=0.06 \mathrm{dBHz} / \mathrm{deg}\end{array}$ & $1.28 \mathrm{dBHz}$ \\
\cline { 2 - 6 } & 2-freq. & S1C, S2P & $\begin{array}{l}a=18.32 \mathrm{dBHz} \\
b=-0.24 \mathrm{dBHz} / \mathrm{deg} \\
c=13.10^{-4} \mathrm{dBHz} / \mathrm{deg}^{2}\end{array}$ & $1.62 \mathrm{dBHz}$ \\
\hline \multirow{3}{*}{ GLONASS } & & $\begin{array}{l}a=2.49 \mathrm{dBHz} \\
b=-0.04 \mathrm{dBHz} / \mathrm{deg}^{-4} \\
c=-2.10^{-4} \mathrm{dBHz} / \mathrm{deg}^{2}\end{array}$ & $1.04 \mathrm{dBHz}$ \\
\hline
\end{tabular}

\subsection{Finding thresholds for detection statistic}

Detection statistic $S_{a}^{S}$ computed by relations (2) and (3) was used to compute threshold $T$ for multipath detection. Similar to $\Delta \hat{C}$ function, also $S_{a}^{S}$ is elevation dependent, probably due to non-linear antenna gain pattern. In (Strode and Groves, 
2016 ) is threshold $T$ computed as a function by fitting elevation dependent $3^{\text {rd }}$ order polynomial to $S_{a}^{S}$ values and then added $t=1,2$ and 3 -fold multiplication of fit RMS $\hat{\sigma}_{S}$. These three levels of threshold serve as multipath severity assessment. After application of this approach to calibration data, more than $3 \%$ of detection statistic $S_{a}^{s}$ values were out of the most strict three-fold threshold function. We decided to edit threshold function by adding multiplicative elevation weighting function according to (4). Exponential function has been chosen as base function with unity weight in zenith and maximal weight equal to $e$ at elevation equal to elevation mask angle $\theta_{\text {cut }}=10$ degree).

$$
T_{t \omega \sigma}(\theta)=\hat{S}_{a}^{S}(\theta)+\underbrace{\alpha \cdot \exp \left(\frac{90^{\circ}-\theta}{90^{\circ}-\theta_{c u t}}\right)}_{\omega(\theta)} \cdot t \cdot \hat{\sigma}_{s}
$$

Proposed weighting function $\omega(\theta)$ is formed by $\alpha$ factor, which has been set empirically with condition that only $0.1 \%$ of all data is greater than three-fold threshold function values. Computed detection statistics for all approaches are shown on Figure 5 along with estimated linear function $\hat{S}_{a}^{s}(\theta)$ (yellow) and 1,2 and 3-fold weighting detection threshold. Coefficient of estimated linear function and empirical $\alpha$ factors are listed in Table 2.
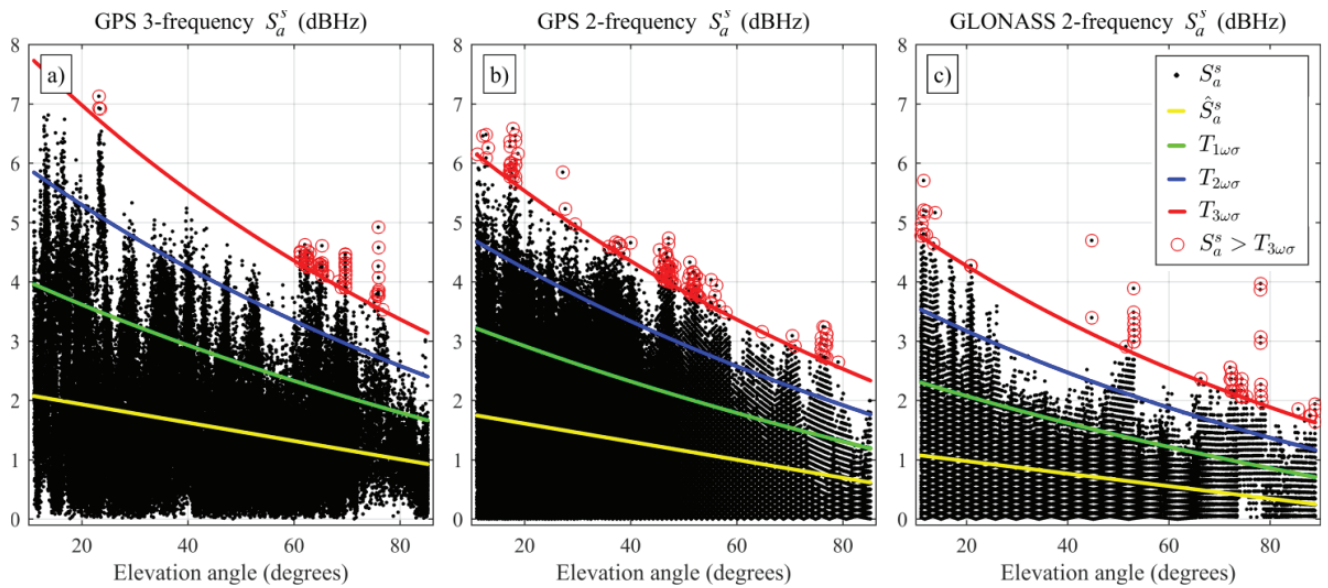

Fig. 5. Computed detection statistic $S_{a}^{S}$ in $\mathrm{dBHz}$ for a) GPS 3-frequency approach (values from Fig. 4a, Fig. 4b were used, b) GPS 2-frequency approach,

c) GLONASS 2-frequency approach 
Table 2. Estimated coefficients of linear fit function $\hat{S}_{a}^{S}(\theta)=a+b \theta$ (on Fig. 5 it is yellow one), standard deviation of this fit $\hat{\sigma}_{S}$ and values of $\alpha$ factors for investigated approach.

\begin{tabular}{|l|l|l|l|l|}
\hline System & Approach & Coefficients of $\hat{S}_{a}^{s}$ & $S_{a}^{s}$ fit RMS $\left(\hat{\sigma}_{s}\right)$ & $\alpha$ factor \\
\hline \multirow{3}{*}{ GPS } & 3-freq. & $\begin{array}{l}a=2.24 \mathrm{dBHz} \\
b=-0.015 \mathrm{dBHz} / \mathrm{deg}\end{array}$ & $0.98 \mathrm{dBHz}$ & 0.71 \\
\cline { 2 - 5 } & 2-freq. & $\begin{array}{l}a=1.91 \mathrm{dBHz} \\
b=-0.015 \mathrm{dBHz} / \mathrm{deg}\end{array}$ & $0.93 \mathrm{dBHz}$ & 0.58 \\
\hline GLONASS & 2-freq. & $\begin{array}{l}a=1.19 \mathrm{dBHz} \\
b=-0.011 \mathrm{dBHz} / \mathrm{deg}\end{array}$ & $0.64 \mathrm{dBHz}$ & 0.70 \\
\hline
\end{tabular}

\section{Multipath detection in urban environment}

To test introduced method of multipath detection we made an experimental GNSS static measurement in urban environment. As a test site we chose Slovak University of Technology principal residence. There is the open courtyard inside building block as it is shown on Fig. 6. Big walls of surrounding, up to $25 \mathrm{~m}$ high buildings, should introduce many reflections. We used the same equipment as during calibration measurement. Data were logging at $1 \mathrm{~s}$ sampling rate for 16 hours on 26 April, 2017.
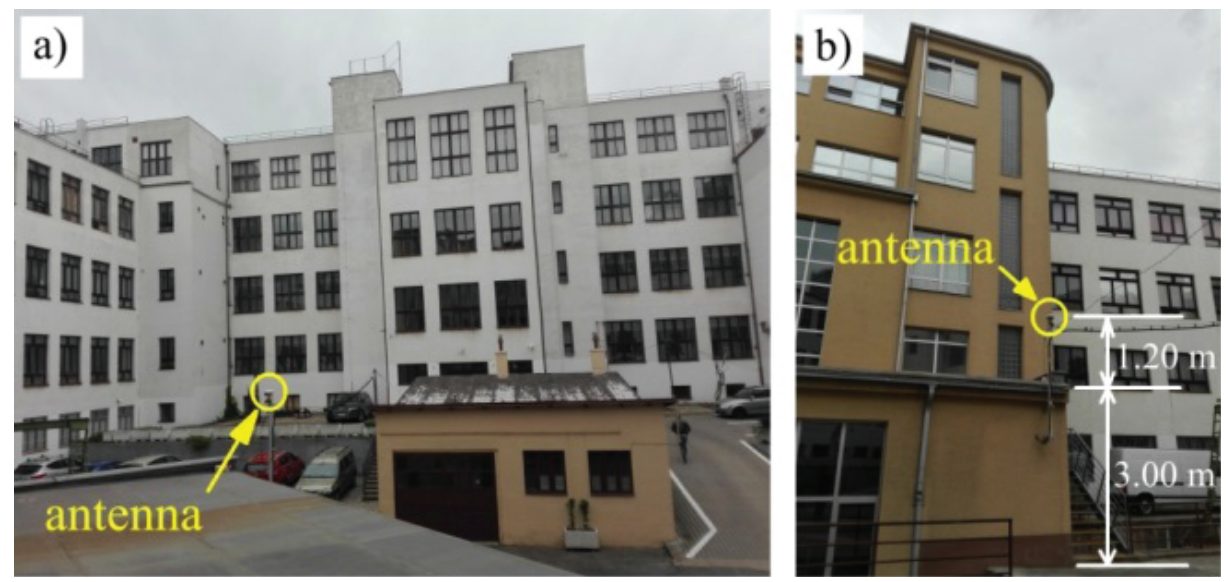

Fig. 6. Experimental site at SUT principal residence. Antenna was placed on the steel rod approximately $4.20 \mathrm{~m}$ above ground ( $1.20 \mathrm{~m}$ above reduced roof) and 5 meters off the nearest wall. The South a) and North-East view b) at the site. 
For measured data detection statistics $S_{a}^{S}$ for each epoch and each tracked satellite were computed according to equation (2) or (3). To find out multipath infected signals we have compared detection statistic values to the most strict threshold function value (according to (4) with $t=3$ ). If the statistic is greater than threshold function value then we consider tested signal in given epoch as multipath. As the reference for verification purposes we used zenith-pointing image panorama taken at site. Panorama was created from the series of images with given orientation in open-source photo stitching software Hugin (http://hugin.sourceforge.net). Proper level and orientating to geographic north was ensured during photographing using the survey tribrach.

It is obvious that if there is tracked signal of satellite which is located "inside" building on the panorama then this signal has to pass by one or more reflection from building walls. In case that the satellite signal is tracked close to building roof edge, it is possible that the signal was diffracted. In both cases, this signal would be corrupted and should be identified by detection. Test results for all three approaches: a) GPS 3-frequency, b) GPS 2-frequency, c) GLONASS 2-frequency, can be seen on skyplot graphic on Fig. 7. By blue color is drawn satellite position with complete set of measurements at given epoch and red circles represent detected multipath. Satellite position with incomplete measurement epochs (e.g. only L1 measurement for GPS) are not shown on the figure. Detection was evaluated only by view. Each of the approach is summarized as following:

a) GPS 3-frequency - only 12 tracked satellites of GPS Block II-F was used for multipath detection. In west and north-west part of horizon almost all signals coming from the building are detected correctly, however in south and southeast part there is many undetected signals coming from building's shadowed areas.

b) GPS 2-frequency - totally 30 satellites with two frequency measurements were tested. For some GPS Block II-F satellites this approach provides slightly better results (G10, G24 and G27) which were tracked in the south part of horizon. This approach does not correctly detected signals from satellites in the south-west part of horizon.

c) GLONASS 2-frequency - GLONASS signals are tracked also in the most shadowed areas in the north part of horizon. Detection of these undesirable signals is very well in this part, almost all in building signals are detected correctly. In the north-west part of horizon the detection is less effective. 



Fig. 7. Skyplot graphics of test measurement multipath detection results. Detection for three approaches was performed: a) GPS 3-frequency, b) GPS 2-frequency and c) GLONASS 2-frequency. Blue dots represent satellite positions with complete set of measurements, red circles are detected multipath events.

\section{Summary and conclusions}

The paper focuses on the possibility of detection multipath effect in GNSS measurements using recorded SNR measurements. This work follows on the previous research of Strode and Groves, mainly demonstrated in (Strode and Groves, 2016). Presented paper introduce simplified version of detection statistic (3) using only two frequencies, which allows also exploitation of other GNSS. Special form of calibration measurement in low-multipath environment has to be done prior to actual use. Originally proposed threshold function to identify multipath infected signals relies on simple three-sigma rule of fitted polynomial function (described in section 2.2). According to unsatisfactory results we obtained by this strategy we propose to use threshold function in form of equation (4) which involves exponential elevation 
weighting function. Discrepancy in our results is unclear, but it may reflect deployment of various equipment (we use receiver of Trimble company, while in original article they use Leica receiver). For testing purposes of two and three frequency detection approaches for GPS and GLONASS systems we made an experimental measurement in urban environment. Comparison between two and three frequency approach is slightly in favor of simplified two frequency approach, but further investigation should be performed. Even though all approaches detected reflected signals from blocked satellites relatively well, none of them detect significant multipath in case of unobstructed view. To improve the sensitivity of the method we think that calibration measurement in different environment should be performed, with conditions more similar to supposed use of equipment (e.g. for urban environment empty concrete parking lot could be used). Utility of another calibration environment will be studied further in the following research.

\section{Acknowledgement}

The authors would like to thank for financial assistance from the Slovak University of Technology in Bratislava, grant scheme for Support of Young Researchers in the project "Multipath Protection of GNSS Station". This work was supported by the Grants No. 1/0682/16 of the Grant Agency of Slovak Republic VEGA.

\section{References}

Global Positioning Systems Directorate System Engineering \& Integration, Interface Control Document Working Group. (2013). Interface Specification IS-GPS-200. Available online: www.gps.gov/ technical/icwg/IS-GPS-200H.pdf

Hofmann-Wellenhof, B., Lichtenegger H., and Wasle, L. (2008). GNSS - Global Navigation Satellite Systems. First edition. Springer-Verlag Wien. ISBN 978-3-211-73012-6.

Lau, L. and Cross, P. (2006). A New Signal-to-Noise-Ratio Based Stochastic Model for GNSS HighPrecision Carrier Phase Data Processing Algorithms in the Presence of Multipath Errors. In: ION GNSS 2006. September 26-29 2006. Forth Worth, Texas.

Lau, L. and Cross, P. (2007). Development and testing of a new ray-tracing approach to GNSS carrierphase multipath modelling. Journal of Geodesy, 81, 713-732. DOI 10.1007/s00190-007-0139-z

Luo, X. (2013). GPS Stochastic Modelling, Signal Quality Measures and ARMA Processes. Doctoral Thesis. Karlsruhe Institute of Technology, Karlsruhe, Germany.

Richards, J.A. (2008). Radio Wave Propagation. An Introduction for the Non-Specialist. Springer-Verlag Berlin.

Rost, Ch. and Wanninger, L. (2009). Carrier phase multipath mitigation based on GNSS signal quality measurements. Journal of Applied Geodesy, 3, 1-8. DOI: 10.1515/JAG.2009.009.

Strode, P.R.R. and Groves, P.D. (2016). GNSS multipath detection using three-frequency signal-to-noise measurements. GPS Solutions, 20, 399-412. DOI: 10.1007/s10291-015-0449-1. 
